\title{
Evaluation of longitudinal and transverse dispersivities/distance ratios for tracer test in a radially convergent flow field with scale-dependent dispersion
}

\author{
Jui-Sheng Chen ${ }^{\text {a }}$, Chen-Wuing Liu ${ }^{\mathrm{b}, *}$, Ching-Ping Liang ${ }^{\mathrm{c}}$ \\ a Institute of Applied Geology, National Central University, Taoyuan 320, Taiwan, ROC \\ ${ }^{\mathrm{b}}$ Department of Bioenvironmental Systems Engineering, National Taiwan University, No. 1, Sec. 4, \\ Roosevelt Road, Taipei 106, Taiwan, ROC \\ ${ }^{\mathrm{c}}$ Department of Environmental Engineering and Sanitation, Foo-Yin University, Kaohsiung 831, Taiwan, ROC
}

Received 22 February 2005; received in revised form 29 July 2005; accepted 2 August 2005

Available online 5 October 2005

\begin{abstract}
This study presents a novel mathematical model for analysis of non-axisymmetrical solute transport in a radially convergent flow field with scale-dependent dispersion. A two-dimensional, scale-dependent advection-dispersion equation in cylindrical coordinates is derived based on assuming that the longitudinal and transverse dispersivities increase linearly with the distance of the solute transported from its injected source. The Laplace transform finite difference technique is applied to solve the two-dimensional, scale-dependent advection-dispersion equation with variable-dependent coefficients. Concentration contours for different times, breakthrough curves of average concentration over concentric circles with a fixed radial distance, and breakthrough curves of concentration at a fixed observation point obtained using the scale-dependent dispersivity model are compared with those from the constant dispersivity model. The salient features of scale-dependent dispersion are illustrated during the non-axisymmetrical transport from the injection well into extraction well in a convergent flow field. Numerical tests show that the scale-dependent dispersivity model predicts smaller spreading than the constant-dispersivity model near the source. The results also show that the constant dispersivity model can produce breakthrough curves of averaged concentration over concentric circles with the same shape as those from the proposed scale-dependent dispersivity model at observation point near the extraction well. Far from the extracting well, the two models predict concentration contours with significantly different shapes. The breakthrough curves at observation point near the injection well from constant dispersivity model always produce lesser overall transverse dispersion than those from scale-dependent dispersivity model. Erroneous dimensionless transverse/longitudinal dispersivity ratio may result from parametric techniques which assume a constant dispersivity if the dispersion process is characterized by a distance-dependent dispersivity relationship. A curve-fitting method with an example is proposed to evaluate longitudinal and transverse scale-proportional factors of a field with scale-dependent dispersion.
\end{abstract}

(c) 2005 Elsevier Ltd. All rights reserved.

Keywords: Scale-dependent dispersion; Non-axisymmetrical solute transport; Laplace transform finite-difference method; Tracer test

\footnotetext{
* Corresponding author. Tel.: +886 2 23626480; fax: +886 2 23639557.

E-mail address: 1cw@gwater.agec.ntu.edu.tw (C.-W. Liu).
}

\section{Introduction}

The migration of dissolved contaminant plume in a subsurface environment is traditionally modeled with the advection-dispersion equation. Most mathematical models for predicting solute transport are based on 
advection-dispersion equation with constant dispersivity. However, both field and laboratory experiments indicate that dispersivity generally increases with solute transport distance. The increase of dispersivity with travel distance results from the heterogeneous nature of porous media. The spatial variability of dispersivity is generally assumed as scale-dependent dispersion $[1,3,5$, $6,8,10,13-15,18,19]$. Researchers have applied numerical solutions $[8,11,14]$ and analytical solutions $[10,13,18,19]$ to investigate scale-dependent dispersive transport in a uniform flow field. Few researchers have developed solutions to two-dimensional advection-dispersion equation with scale-dependent dispersion in a uniform flow field. Hunt [6] developed one-, two- and three-dimensional analytical solution for a scale-dependent dispersion equation. Alternatively, Aral and Liao [1] developed analytical solutions for two-dimensional advection-dispersion equation with a time-dependent dispersion coefficient.

The advection-dispersion equation in cylindrical coordinates refers to the problem of analyzing the dispersive transport of a contaminant in the radial flow field generated by extraction or injection well that fully penetrates a homogeneous confined aquifer. Current contaminant transport models in a radial flow field are also based on advection-dispersion equation with constant dispersivity. Relatively a few studies have developed solutions to advection-dispersion equations in cylindrical coordinates to investigate how scale-dependent dispersion affects solute transport in a radial flow field. Kocabas and Islam [9] developed solution to radial advection-dispersion equation assuming spatially velocity and scale dependence of dispersivity for a radially divergent flow field. Chen et al. [3] presented a semi-analytical solution for describing the transport of dissolved substance in a radially convergent flow field with linearly scale-dependent dispersion. Their solution was applied to a set of reported field data to examine its applicability. The application results reveal that the solute transport process at the test site obeys the linearly scale-dependent dispersion model.

To our knowledge, no research works focus on the development of the solution to two-dimensional, scale dependent advection-dispersion equation in cylindrical coordinates for describing non-axisymmertrical solute transport in a convergent flow field. Solutions to two-dimensional, scale-dependent advection-dispersion equation in cylindrical coordinates help predicting breakthrough curves at observation points. If function relationship between the distance and dispersivity obeys the scale-dependent dispersion assumption, field concentration breakthrough curves can be fitted against the type curve from the developed model to obtain transverse scale-related transport parameter. In light of the above development, this study presents a two-dimensional mathematical model to investigate the effect of scale-dependent dispersion on non-axisymmetrical solute transport in a radially convergent flow field. Additionally, a graphical method with an example, which applied a curve-fitting procedure involving type curves at the extraction well and at one observation point, is proposed to determine the scale-dependent longitudinal and transverse dispersivity for a tracer test.

\section{Governing equation}

To investigate non-axisymmetrical solute transport in a radially convergent flow field with scale-dependent dispersion, the problem of a convergent tracer test is considered herein. Fig. 1 presents the conceptual configuration. The governing equations and boundary conditions are derived and described below.

The radially convergent flow field is generated by a vertically oriented extraction well of radius $r_{\mathrm{w}}$ located at $r=0$, and fluid pumped at a constant volume rate of $Q$ from a statistically homogeneous and isotropic aquifer of infinite horizontal extent. Assuming that the tracer injection has no influence on the flow field, therefore the mean pore velocity $V$ caused by extraction is described by

$V=-\frac{A}{r}$

where $A=\frac{Q}{2 \pi b \phi}$, and $b$ and $\phi$ represent the aquifer thickness and effective porosity.

The two-dimensional, non-axisymmetrical solute transport in a radial flow field can be described by the

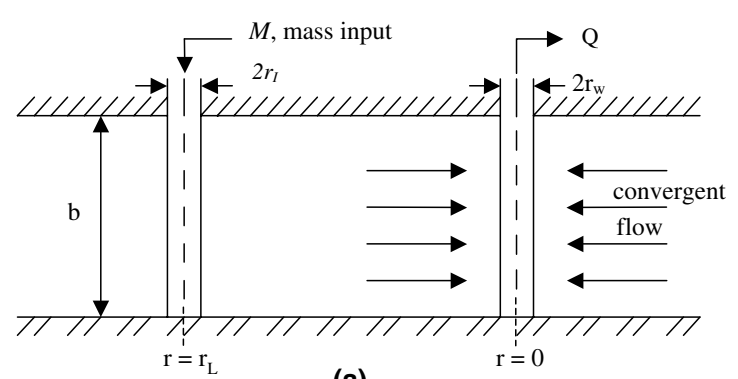

(a)

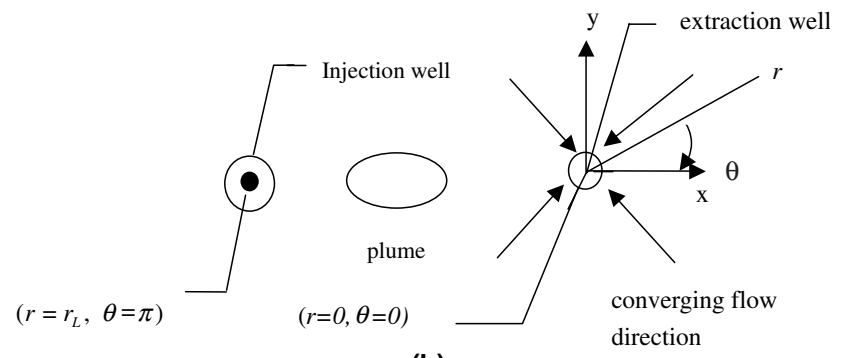

(b)

Fig. 1. Schematic diagram of radically convergent tracer test: (a) side view and (b) plane view. 
advection-dispersion equation in cylindrical coordinates as

$R \frac{\partial C}{\partial t}=\frac{1}{r} \frac{\partial}{\partial r}\left(r D_{\mathrm{L}} \frac{\partial C}{\partial r}\right)-V \frac{\partial C}{\partial r}+\frac{1}{r^{2}} \frac{\partial}{\partial \theta}\left(D_{\mathrm{T}} \frac{\partial C}{\partial \theta}\right)$

where $C$ is the concentration, $D_{\mathrm{L}}$ and $D_{\mathrm{T}}$ denote longitudinal and transverse dispersion coefficients, respectively, and $R$ is retardation factor.

The longitudinal and transverse dispersion coefficients, $D_{\mathrm{L}}$ and $D_{\mathrm{T}}$, in Eq. (2) are generally considered to be a linear function of the mean pore velocity and molecular diffusion can be neglected as follows:

$D_{\mathrm{L}}=a_{\mathrm{L}}|V|$

$D_{\mathrm{T}}=a_{\mathrm{T}}|V|$

where $a_{\mathrm{L}}$ and $a_{\mathrm{T}}$ are longitudinal and transverse dispersivities, respectively. For the constant longitudinal and transverse dispersivities, $a_{\mathrm{L}}=a_{\mathrm{L}, \mathrm{c}}$ and $a_{\mathrm{T}}=a_{\mathrm{T}, \mathrm{c}}$, Eqs. (2) and (3) reduce to the classical, two-dimensional advection-dispersion equation in cylindrical coordinates [2]

$R \frac{\partial C}{\partial t}=\frac{a_{\mathrm{L}, \mathrm{c}} A}{r} \frac{\partial^{2} C}{\partial r^{2}}+\frac{A}{r} \frac{\partial C}{\partial r}+\frac{a_{\mathrm{T}, \mathrm{c}} A}{r^{3}} \frac{\partial^{2} C}{\partial \theta^{2}}$

Results from field and laboratory experiments suggested that both longitudinal and transverse dispersivities may be scale-dependent, i.e., $a_{\mathrm{L}}$ and $a_{\mathrm{T}}$ increase with travel distance from the contaminant source. It is assumed that any growth with travel distance of the dispersion process is a direct consequence of the heterogeneous nature of the porous medium [18]. In this paper, the growth of longitudinal dispersivity, $a_{\mathrm{L}, \mathrm{s}}$ as a linear function of travel distance from the injection well located at $r=r_{\mathrm{L}}$ is employed herein and can be adequately described using equations of the form [14]

$a_{\mathrm{L}, \mathrm{s}}=a_{\mathrm{L}}(r)=e_{\mathrm{L}}\left(r_{\mathrm{L}}-r\right)$

The relationship between the scale-dependent dispersivities in longitudinal and transverse directions may be assumed to be constant as that in constant dispersivity model (CDM) [1,7,8]. Several researchers including Hunt [6,7], Pang and Hunt [13], and Jayawardena and Lui [8], Yates [19] extended the relationships to describe the scale dependent transverse dispersivity in the development of analytical solutions or numerical solutions of solute transport through groundwater system. In this study, a linear empirical relationship was adopted to describe the scale-dependent traverse dispersivity. The transverse dispersivity $a_{\mathrm{T}, \mathrm{s}}$ can be adequately described in the following form:

$a_{\mathrm{T}, \mathrm{s}}=a_{\mathrm{T}}(r)=X_{\mathrm{s}} e_{\mathrm{L}}\left(r_{\mathrm{L}}-r\right)=e_{\mathrm{T}}\left(r_{\mathrm{L}}-r\right)$

where $e_{\mathrm{L}}$ and $e_{\mathrm{T}}$ are longitudinal and transverse dispersivity/distance ratios (i.e., scale-proportional factors), respectively, which characterize the scale-dependent dispersion process. $X_{\mathrm{S}}=\frac{a_{\mathrm{T}, \mathrm{s}}}{a_{\mathrm{L}, \mathrm{s}}}=\frac{a_{\mathrm{T}}(r)}{a_{\mathrm{L}}(r)}$ and $X_{\mathrm{c}}=\frac{a_{\mathrm{T}, \mathrm{c}}}{a_{\mathrm{L}, \mathrm{c}}}$ are dimensionless dispersivity ratios for SDM and CDM, respectively. Notice that $a_{\mathrm{L}}$ and $a_{\mathrm{T}}$ are both zero at the tracer injection well $r=r_{\mathrm{L}}$ and reach the maximum at the extraction well $r=r_{\mathrm{L}}$.

Substituting Eqs. (1), (3a), (3b), (5a) and (5b) into Eq. (2) yields the following equation:

$$
\begin{aligned}
R \frac{\partial C}{\partial t}= & \frac{1}{r} \frac{\partial}{\partial r}\left(r e_{\mathrm{L}}\left(r_{\mathrm{L}}-r\right) \frac{A}{r} \frac{\partial C}{\partial r}\right)+\frac{A}{r} \frac{\partial C}{\partial r} \\
& +\frac{1}{r^{2}} \frac{\partial}{\partial \theta}\left(e_{\mathrm{T}}\left(r_{\mathrm{L}}-r\right) \frac{A}{r} \frac{\partial C}{\partial \theta}\right)
\end{aligned}
$$

Rewriting Eq. (6) in more clearly visible form as

$$
\begin{aligned}
R \frac{\partial C}{\partial t}= & \frac{e_{\mathrm{L}} A}{r}\left(r_{\mathrm{L}}-r\right) \frac{\partial^{2} C}{\partial^{2} r}+\frac{A\left(1-e_{\mathrm{L}}\right)}{r} \frac{\partial C}{\partial r} \\
& +\frac{e_{\mathrm{T}} A\left(r_{\mathrm{L}}-r\right)}{r^{3}} \frac{\partial^{2} C}{\partial \theta^{2}}
\end{aligned}
$$

The advective transport term of Eq. (7) becomes negative when $e_{\mathrm{L}}>1$, therefore would cause an apparent negative advective transport. It seems improbable that effect of the upstream dispersion is so strong and greater than that of advective transport during the growth of dispersion with the solute displacement distance. Based on the impracticability of negative advective transport and considering available field evidence, the $e_{\mathrm{L}}$ should be restricted to the range $0<e_{\mathrm{L}}<1$.

The aquifer's initial tracer concentration is assumed to be zero before starting the test:

$C(r, \theta, t=0)=0$

The boundary, which is used to describe solute transport at the interface between the extraction well and aquifer while neglecting the finite volume in the well bore is as

$\frac{\partial C(r, \theta, t)}{\partial r}=0 \quad$ at $r=r_{\mathrm{w}}$

Because the concentration is symmetrical across $\theta=0$ and $\theta=\pi$, only upper half domain is considered for mathematical simplicity. The boundary condition including solute transport at the injection well boundary can be formulated as [20]

$$
C(r, \theta, t)= \begin{cases}C_{\mathrm{I}}(t) & \pi-\delta<|\theta|<\pi \\ 0 & 0<|\theta|<\pi-\delta\end{cases}
$$

where $C_{\mathrm{I}}(t)$ is the concentration generated in the injection well and transported downstream the narrow and short (a few well diameters) discharge zone by advection. This small zone with advection-dominated flow has an aperture angle of $2 \delta$ (Fig. 2). The aperture angle of this narrow zone at the distance $r^{*} \approx r_{\mathrm{L}}-l\left(l \ll r_{\mathrm{L}}\right)$ from the center of extraction is

$2 \delta=\frac{2 \alpha r_{\mathrm{I}}}{r_{\mathrm{L}}}$ 


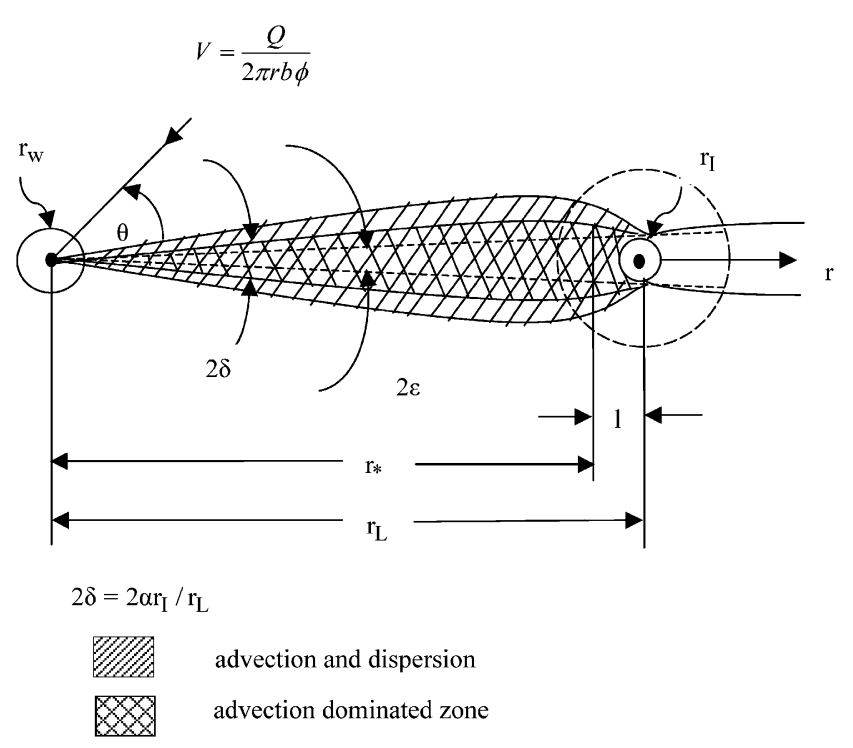

Fig. 2. Plane view of the injection well boundary condition (after Zlotnik and Logan [20]).

where $r_{\mathrm{I}}$ represents the injection well radius, $\alpha$ is a factor that defines the distortion of distance between the two most separated streaming line entering (or leaving) the injection well (this parameter can also depend on skin effect for an injection well, see Zlotnik and Logan [20]; Eq. (3)).

The effluent concentration from the injection well in an ambient horizontal flow is yet to be determined. The effluent concentration from the well with initial dissolved tracer mass, $M_{0}$, satisfies a mass balance equation for the tracer in borehole:

$$
\begin{aligned}
& -2 \alpha r_{\mathrm{I}} \phi b\left|V\left(r_{\mathrm{L}}\right)\right| C_{\mathrm{I}}(t)=\pi r_{\mathrm{I}}^{2} h_{\mathrm{I}} \frac{\mathrm{d} C_{\mathrm{I}}(t)}{\mathrm{d} t} \\
& C_{\mathrm{I}}(t=0)=\frac{M_{0}}{\pi r_{\mathrm{I}}^{2} h_{\mathrm{I}}}=C_{0}
\end{aligned}
$$

where $h_{\mathrm{I}}$ is the mixing length of injection well [20, Eq. (6)].

After integration, the known effluent concentration $C_{\mathrm{I}}(t)$ can be substituted into boundary condition (10).

The physics of the problem stipulates that $C$ is a single-valued function in $r$ and $\theta$ coordinates. In addition, $C$ is continuous and symmetrical function across $\theta=0$ and $\theta=\pi$. Thus, boundary conditions in the transverse directions are as follows:

$\frac{\partial C(r, 0, t)}{\partial \theta}=0$
$\frac{\partial C(r, \pi, t)}{\partial \theta}=0$

Dimensionless variables are defined in a manner similar to that used by Chen et al. [3]. Following Moench [12] and substituting the definition given in Table 1 into Eq. (7), the dimensionless transport equation is presented in the following form:
Table 1

Dimensionless parameters used in this study

\begin{tabular}{ll}
\hline Dimensionless quantity & Expression \\
\hline Time & $t_{\mathrm{D}}=\frac{Q t}{\pi b \phi\left(r_{\mathrm{L}}^{2}-r_{\mathrm{w}}^{2}\right)}$ \\
Distance & $r_{\mathrm{D}}=\frac{r}{r_{\mathrm{L}}}$ \\
Extraction well radius & $r_{\mathrm{wD}}=\frac{r_{\mathrm{w}}}{r_{\mathrm{L}}}$ \\
Transverse scale-proportional factor & $X_{e}=\frac{e_{\mathrm{T}}}{e_{\mathrm{L}}}$ \\
Extraction well mixing factor & $\mu_{\mathrm{w}}=\frac{r_{\mathrm{w}}^{2} h_{\mathrm{w}}}{\phi h_{\mathrm{w}}\left(r_{\mathrm{L}}^{2}-r_{\mathrm{w}}^{2}\right)}$ \\
Injection well mixing factor & $\mu_{\mathrm{I}}=\frac{r_{\mathrm{I}} r_{\mathrm{L}} h_{\mathrm{I}}}{\phi h_{\mathrm{I}}\left(r_{\mathrm{L}}^{2}-r_{\mathrm{w}}^{2}\right)}$ \\
\hline
\end{tabular}

$$
\begin{aligned}
& e_{\mathrm{L}}\left(1-r_{\mathrm{D}}\right) \frac{\partial^{2} C}{\partial r_{\mathrm{D}}^{2}}+\left(1-e_{\mathrm{L}}\right) \frac{\partial C}{\partial r_{\mathrm{D}}}+\frac{e_{\mathrm{T}}\left(1-r_{\mathrm{D}}\right)}{r_{\mathrm{D}}^{2}} \frac{\partial^{2} C}{\partial \theta^{2}} \\
& =\frac{2 r_{\mathrm{D}} R}{\left(1-r_{\mathrm{wD}}^{2}\right)} \frac{\partial C}{\partial t_{\mathrm{D}}}
\end{aligned}
$$

Consequently, the initial and boundary conditions (9)-(15) become

$$
\begin{aligned}
& C\left(r_{\mathrm{D}}, \theta, 0\right)=0 \\
& \frac{\partial C\left(r_{\mathrm{D}}, \theta, t_{\mathrm{D}}\right)}{\partial r_{\mathrm{D}}}=0 \quad \text { at } r_{\mathrm{D}}=r_{\mathrm{wD}} \\
& C\left(r_{\mathrm{D}}, \theta, t_{\mathrm{D}}\right)= \begin{cases}C_{\mathrm{I}}\left(t_{\mathrm{D}}\right) & \pi-\delta<|\theta|<\pi \\
0 & 0<|\theta|<\pi-\delta\end{cases} \\
& -C_{\mathrm{I}}\left(t_{\mathrm{D}}\right)=\mu_{\mathrm{I}} \frac{\mathrm{d} C_{\mathrm{I}}\left(t_{\mathrm{D}}\right)}{\mathrm{d} t_{\mathrm{D}}} \\
& C_{\mathrm{I}}\left(t_{\mathrm{D}}=0\right)=C_{0} \\
& \frac{\partial C\left(r_{\mathrm{D}}, \theta, t_{\mathrm{D}}\right)}{\partial \theta}=0 \quad \text { at } \theta=0 \\
& \frac{\partial C\left(r_{\mathrm{D}}, \theta, t_{\mathrm{D}}\right)}{\partial \theta}=0 \quad \text { at } \theta=\pi
\end{aligned}
$$

This study adopts the Laplace transform finite difference, LTFD, technique to solve the initial-boundary value problem specified by Eqs. (16)-(23). Appendix A provides a detailed derivation of LTFD method.

\section{Results and discussion}

\subsection{Model verification}

The one-dimensional Laplace transform analytical solution derived by Chen et al. [3] for simulating the axisymmetrical solute transport behavior in a convergent flow field with scale-dependent dispersion was used to verify the accuracy of the developed LTFD model. Table 2 summaries the simulation conditions and transport parameters in the model verification. Notably, the LTFD model determines the solution analytically in the $\theta$ coordinate and numerically in the $r$ coordinate. An uniform grid mesh was used to discretize the radial distance in the LTFD model. Generally, numerical procedures based on the finite difference methods performed well 
Table 2

Descriptive simulation conditions and transport parameters in the model verification

\begin{tabular}{ll}
\hline Parameter & Tracer test \\
\hline Pumping rate $(Q), \mathrm{m}^{3} \mathrm{~min}^{-1}$ & 2 \\
Aquifer thickness $(h), \mathrm{m}$ & 10 \\
Effective porosity $(\phi)$, dimensionless & 0.2 \\
Radius of extraction well $\left(r_{\mathrm{w}}\right), \mathrm{m}$ & 0.1 \\
Extraction well mixing length $\left(h_{\mathrm{w}}\right), \mathrm{m}$ & 10 \\
Radius of injection well $\left(r_{\mathrm{I}}\right), \mathrm{m}$ & 0.1 \\
Injection well mixing length $\left(h_{\mathrm{I}}\right), \mathrm{m}$ & 10 \\
Distance to the injection well $\left(r_{\mathrm{L}}\right), \mathrm{m}$ & 5 \\
Injected mass $(M)$, kg & 10 \\
Longitudinal dispersivity $\left(a_{\mathrm{L}}\right), \mathrm{m}$ & $1.25,0.25,0.0125$ \\
Peclet number $(P e)$, dimensionless & $1,10,60$ \\
Transverse dispersivity $\left(a_{\mathrm{T}}\right), \mathrm{m}$ & $0.25,0.05,0.0125$ \\
Dimensionless dispersivity ratio & 0.2 \\
$\left(X_{\mathrm{s}}\right.$ or $\left.X_{\mathrm{c}}\right)$, dimensionless & \\
\hline
\end{tabular}

for dispersion-dominated transport (large longitudinal scale-proportional factor), but suffered from excessive artificial oscillation and numerical dispersion when was applied to a advection-dominated transport (small longitudinal scale-proportional factor). The problem of artificial oscillation can be solved by upstream weighting technique. Upstream weighting technique, however, tends to aggravate the numerical dispersion problem. This study uses upstream weighting technique to prevent the artificial oscillation, and employs an extra fine grid mesh to eliminate numerical dispersion.

The grid number $\left(N_{\text {grid }}\right)$ is the total number of grid that is employed to discretize the radial distance using LTFD for simulation of the axisymmetrical solute transport in a convergent flow field. The use of suitable $N_{\text {grid }}$ which required to eliminate numerical dispersion was investigated. Fig. 3 displays breakthrough curves at extraction well for longitudinal scale-proportional factor value of $e_{\mathrm{L}}=1,0.2$ and 0.05 , and compares them to numerical solution developed by Chen et al. [3] for various grid numbers. Two solutions agree closely for a large longitudinal scale-proportional factor of $e_{\mathrm{L}}=$ 1. The LTFD model with a coarse mesh $\left(N_{\text {grid }}=100\right)$ yields noticeable numerical dispersion for $e_{\mathrm{L}}=0.2$. The numerical dispersion becomes significant for $e_{\mathrm{L}}=$ 0.05. The LTFD solutions of the fine grid mesh $\left(N_{\text {grid }}=500\right)$ agree well with analytical solution for $e_{\mathrm{L}}=0.2$, yet the error resulted from the numerical dispersion is noticeable for $e_{\mathrm{L}}=0.05$. By employing the extra fine grid mesh $\left(N_{\text {grid }}=5000\right)$, the LTFD solution agrees closely with the analytical solution for $e_{\mathrm{L}}=$ 0.05 . Increasing the grid numbers reduces and eventually eliminates the numerical dispersion error.

\subsection{Analysis of non-axisymmetrical scale-dependent dispersion transport}

Following validation with analytical solution, the developed model was applied to illustrate non-axisym-

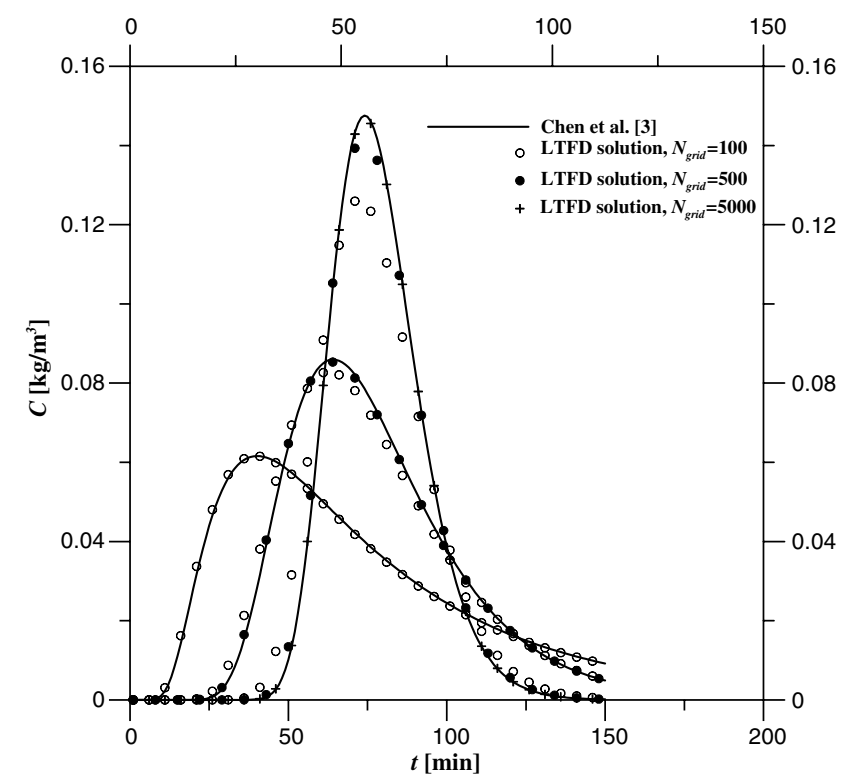

Fig. 3. Comparison of the developed Laplace transform finite-difference (LTFD) method with different grid numbers and Chen's et al. [3] solution for $e_{\mathrm{L}}=1,0.2$ and 0.05 .

metrical transport in a convergent flow field with scale-dependent dispersion. The input parameters are the same as those in the model verification in the Section 3.1. Fig. 4 compares the concentration contour obtained from the scale-dependent dispersivity model (SDM) described herein, where $e_{\mathrm{L}}=1$ and the constant dispersivity model $(\mathrm{CDM})$ that has a constant longitudinal dispersivity value of $a_{\mathrm{L}, \mathrm{c}}=1.25 \mathrm{~m}$ for times equal to 30, 60 and $90 \mathrm{~min}$. The constant longitudinal dispersivity value of $a_{\mathrm{L}, \mathrm{c}}=1.25 \mathrm{~m}$ used in CDM is based on that the correlation $\left(\frac{r_{\mathrm{L}} \cdot e_{\mathrm{L}}}{a_{\mathrm{L}, \mathrm{c}}}=4\right)$ approximated by Chen et al. [3] who fitted the breakthrough curves at extraction well obtained from CDM against the breakthrough curves from SDM. The value of dimensionless dispersivities ratio $\left(X_{\mathrm{s}}=\frac{a_{\mathrm{T}, \mathrm{s}}}{a_{\mathrm{L}, \mathrm{s}}}=\frac{e_{\mathrm{T}}\left(r_{\mathrm{L}}-r\right)}{e_{\mathrm{L}}\left(r_{\mathrm{L}}-r\right)}=\frac{e_{\mathrm{T}}}{e_{\mathrm{L}}}\right.$ or $\left.X_{\mathrm{c}}=\frac{a_{\mathrm{T}, \mathrm{c}}}{a_{\mathrm{L}, \mathrm{c}}}\right)$ is 0.2 in both SDM and CDM. The solid and dashed lines in Fig. 4 represent the concentration contours from CDM and from SDM, respectively. Fig. 4 reveals that a greater spreading of contaminant plume near the injection well predicted by CDM than that obtained using SDM. As the time increases, the development of spreading of contaminant plumes by CDM becomes progressively increasing than that by SDM. However, the two-dimensional concentration contour is the combinational effect of longitudinal and transverse dispersivities which does not clearly differentiate the individual effect of the longitudinal and transverse scale-dependent dispersions on the non-axisymmetrical solute transport in a radially convergent flow filed.

To further examine the individual influence of longitudinal or transverse dispersion on solute transport, the effect of transverse dispersion is excluded first. Because 


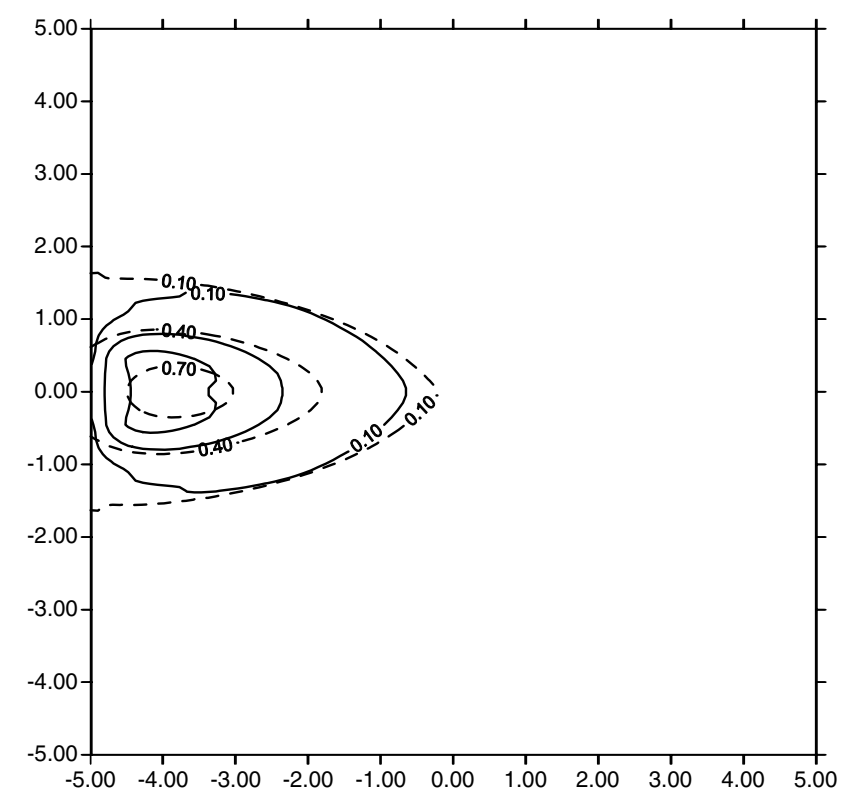

(a)

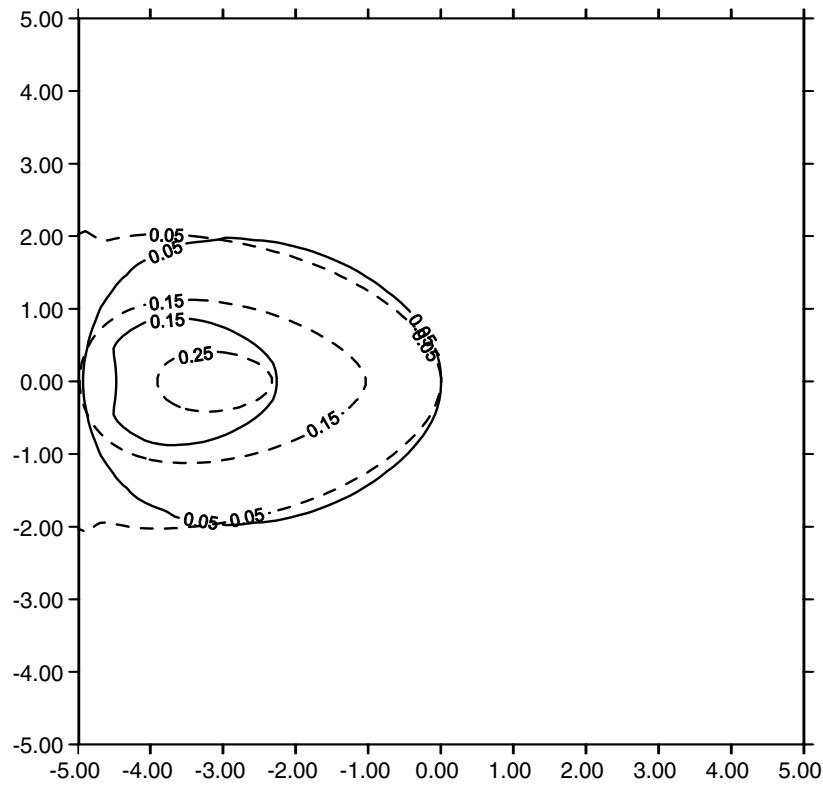

(b)

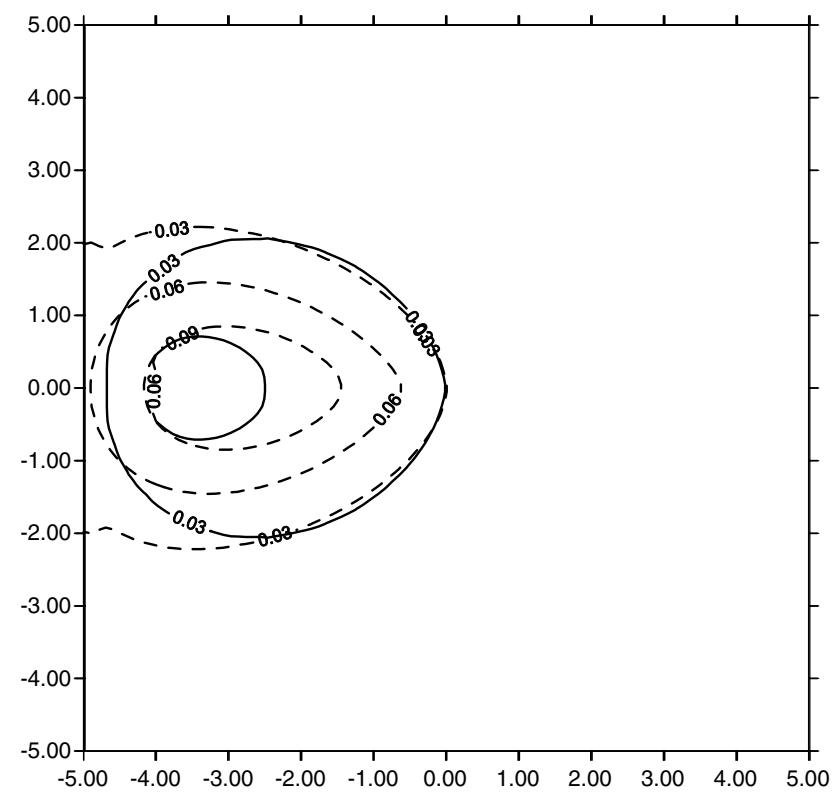

(c)

Fig. 4. Comparison of concentration contours between the scale-dependent dispersivity model (solid line) and the constant dispersivity model (dashed line) in a hypothetical tracer test. (a) $t=30$; (b) $t=60$ and (c) $t=90 \mathrm{~min}$.

transverse scale-dependent dispersion is excluded, the breakthrough curves of average concentration at a fixed radial distance are constructed. The average concentration, $\bar{C}$, is calculated by averaging the solute concentration from transverse angle $\theta=0$ to $\theta=2 \pi$ over concentric circles with a fixed radial distance, $\bar{C}(r, t)=\frac{1}{2 \pi} \int_{0}^{2 \pi} C(r, \theta, t) \mathrm{d} \theta$. The average concentration implies that the influence of transverse dispersion is excluded and only effect of longitudinal dispersion is taken into account.

Fig. 5 displays breakthrough curves of averaging concentration over concentric circles with different radial distance values $(r=0.5,1,2$ and $3 \mathrm{~m})$ from SDM for $e_{\mathrm{L}}=1$ and compares with those from CDM for with constant longitudinal dispersivity value of $a_{\mathrm{L}, \mathrm{c}}=1.25 \mathrm{~m}$. The breakthrough curves obtained from SDM closely match with those using CDM for $r=0.5$ and $1 \mathrm{~m}$, but exhibit significant deviations from those obtained from $\mathrm{CDM}$ for $r=2$ and $3 \mathrm{~m}$. The deviations increase with increasing radial distance. This result is consistent with longitudinal scale-dependent dispersion assumption. Owing to the constant longitudinal dispersivity value used in CDM are derived from breakthrough curves at extraction well (with longer travel distance from 

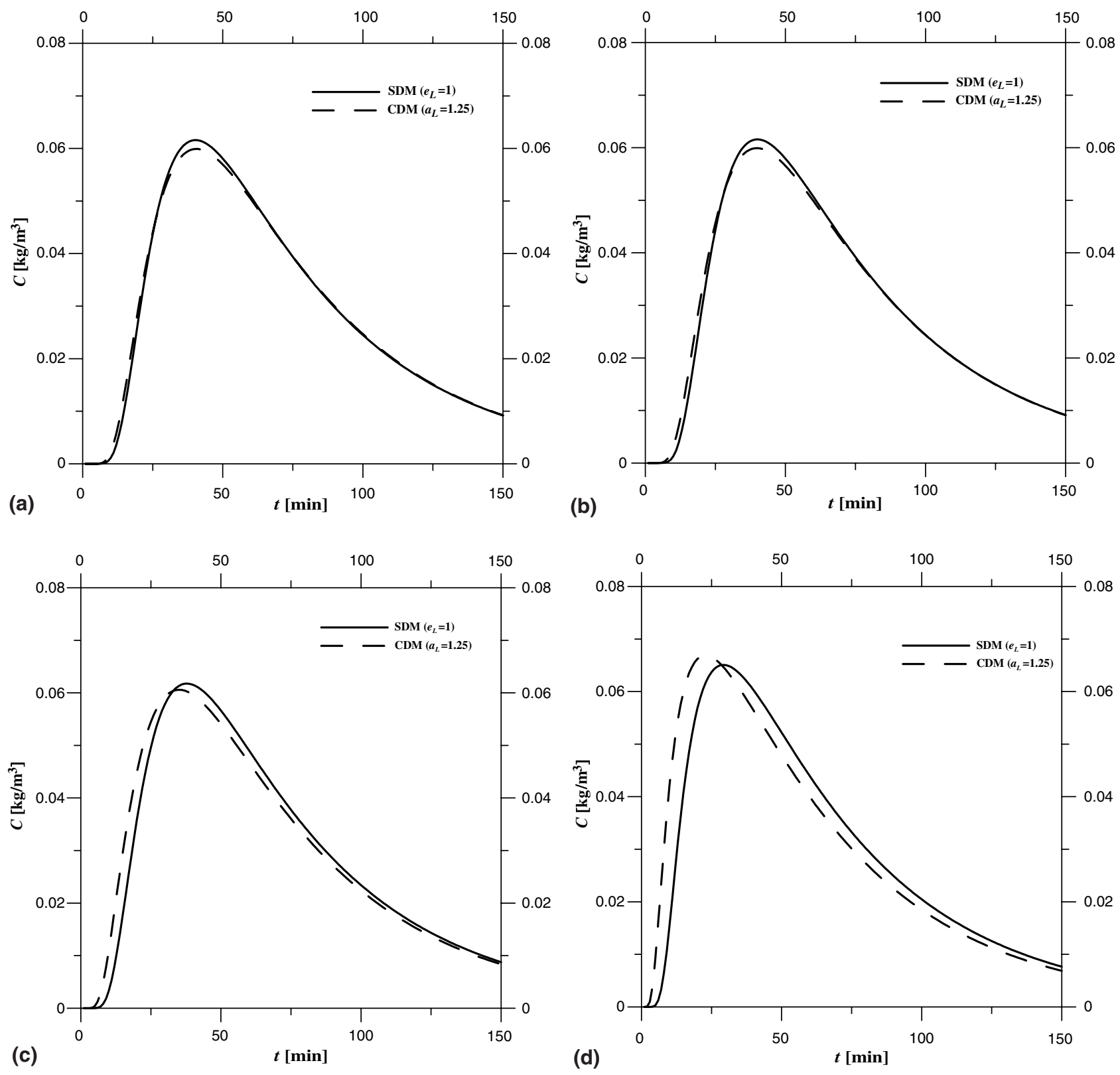

Fig. 5. Comparison of breakthrough curves of average concentration on a concentric circle between the scale-dependent disperivity model (SDM) and the constant dispersivity model (CDM) in a hypothetical tracer test. (a) $r=\frac{1}{10} r_{\mathrm{L}}$; (b) $r=\frac{1}{5} r_{\mathrm{L}}$; (c) $r=\frac{2}{5} r_{\mathrm{L}}$ and (d) $r=\frac{3}{5} r_{\mathrm{L}}$. The average concentrations are calculated by averaging the solute concentration from transverse angle $\theta=0$ to $\theta=\pi$. $\bar{C}(r, t)=\frac{1}{2 \pi} \int_{0}^{2 \pi} C(r, \theta, t) \mathrm{d} \theta$.

injection point), the SDM has a much smaller dispersivity and, therefore, lesser dispersion for larger value of $r$ (with shorter travel distance from injection point) than the CDM, which uses a constant longitudinal dispersivity for the simulation if the dispersion process is characterized with distance-dependent model. Therefore for observation distance at large radial distance (or small travel distance), the CDM model overestimates longitudinal dispersion for the solute transport with scaledependent dispersion. The comparisons of averaging concentration from SDM and CDM have the same trend for $e_{\mathrm{L}}=0.2$ and 0.05 (not shown here).
To more clearly illustrate how the transverse scaledependent dispersion affects the solute transport in a convergent flow field, Fig. 6 compares the breakthrough curves at the observation point $(r=1, \theta=\pi)$ at $e_{\mathrm{L}}=1$, 0.2 and 0.05 . The dispersive front of breakthrough indicates that the CDM yields a higher transverse dispersivity. Additionally, Fig. 7 compares transverse profile at $r=1$ for time equal to 30 [min] from SDM and CDM at $e_{\mathrm{L}}=1$, the transverse concentration profiles of CDM and SDM are both symmetrical around $\theta=\pi$ and the maximum concentrations are at $\theta=\pi$. The $\mathrm{CDM}$ produces a higher maximum concentration and 

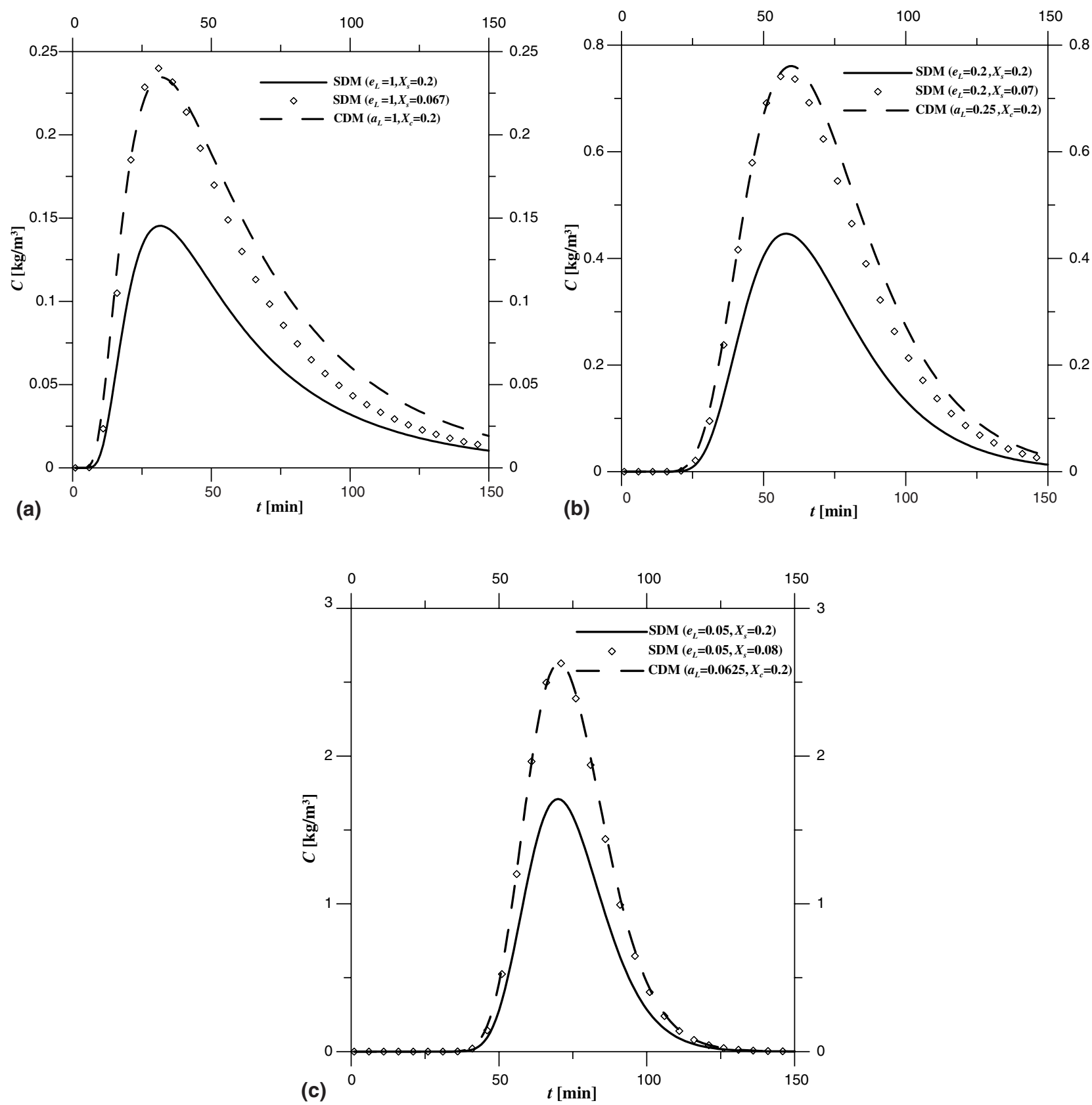

Fig. 6. Comparison of dimensional breakthrough curves at observation point $r=1 / 5 r_{\mathrm{L}}, \theta=\pi$ from the scale-dependent dispersivity model (SDM) and the constant dispersivity model $(\mathrm{CDM})$ for dimensionless transverse/longitudinal dispersivity ratios $X_{\mathrm{s}}$ and $X_{\mathrm{c}}$ with (a) $e_{\mathrm{L}}=1 ;$ (b) $e_{\mathrm{L}}=0.2$ and (c) $e_{\mathrm{L}}=0.05$.

have lesser overall transverse dispersion. The total mass of solute in the concentric circle of $r=1$ is the same both from SDM and CDM because the same breakthrough curves of averaging concentration was used in Fig. 5. This implies CDM model underestimates dimensionless transverse/longitudinal dispersivity ratio for scaledependent transverse dispersion. To provide further evidence of this phenomenon, the input dimensionless transverse/longitudinal dispersivity ratio used in SDM was reduced and breakthrough curves obtained with SDM were fitted against the breakthrough curves for
CDM. The comparison in Fig. 6 reveals that the breakthrough curves obtained with CDM are shaped roughly the same as that those obtained with SDM for rising limbs when the input dimensionless transverse/longitudinal dispersivity ratios in SDM are reduced to 0.067 , 0.7 and 0.8 for $e_{\mathrm{L}}=1,0.2$ and 0.05 . However, a significant discrepancy occurs at the spreading tailing of breakthrough curves between SDM and CDM at $e_{\mathrm{L}}=1$ and 0.2 , the spreading tailing of breakthrough curves between SDM and CDM agree well at $e_{\mathrm{L}}=0.05$. 


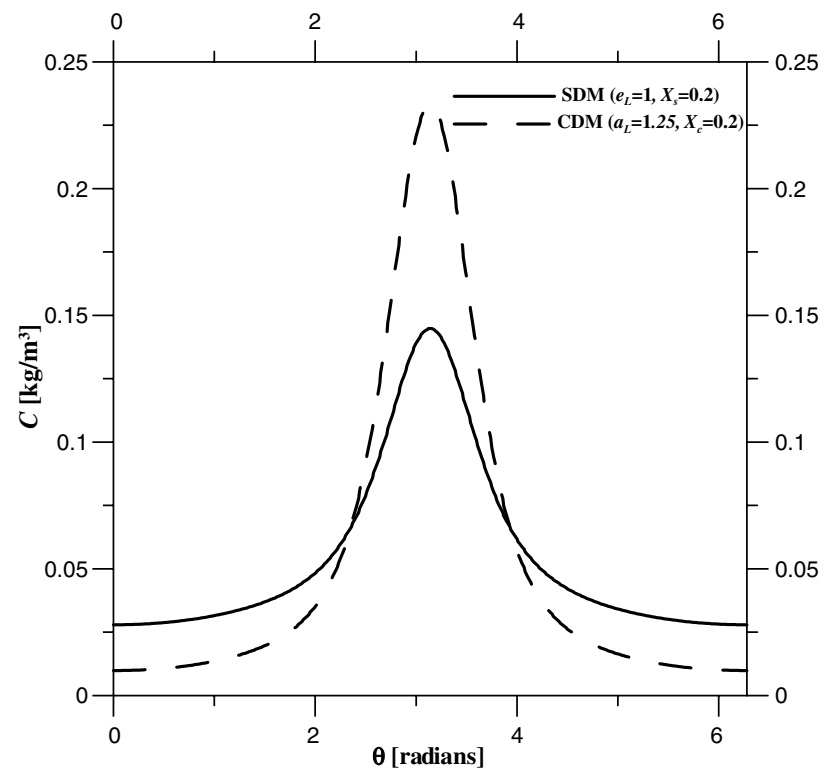

Fig. 7. Comparison of transverse concentration profile at observation $r=\frac{1}{5} r_{\mathrm{L}}$. and $t=30 \mathrm{~min}$ from the scale-dependent dispersivity model (SDM) and the constant dispersivity model (CDM) for dimensionless transverse/longitudinal dispersivity ratios $X_{\mathrm{s}}$ and $X_{\mathrm{c}}$.

From the above comparisons on breakthrough curves and the spatial profile of concentration, erroneous dimensionless transverse/longitudinal dispersivity ratio may be estimated from CDM if the dispersion process is characterized by scale-dependent dispersion.

\subsection{Determination of longitudinal and transverse dispersivity/distance ratios}

Data from the tracer test are commonly analyzed by matching with theoretical breakthrough curves to determine the transport parameters. Chen et al. [2] pointed out that the longitudinal and transverse dispersivities can be simultaneously determined from breakthrough curves at the extraction well and an observation well in a field tracer test with constant dispersivities/distance relationship. In a similar manner the longitudinal and transverse scale-proportional factors can be estimated.

Analysis procedures of the proposed method are outlined as follows:

1. Estimation of the longitudinal scale-proportional factor.

(a) The type curves at the extraction well obtained from the SDM are plotted logarithmically for various longitudinal scale-proportional factors.

(b) The experimental concentrations measured at the extraction well are normalized relative to the peak concentration and are plotted in semi-logarithmically against the real time.

(c) The experimental data are matched against the type curves from the developed SDM and the longitudinal scale-proportional factor is obtained.

2. Estimation of the transverse scale-proportional factor.

(a) The breakthrough curve of experimental concentration observed at other observation well is plotted against real time.

(b) The theoretical breakthrough curves for different dimensionless dispersivity ratio at this observation well are generated from the developed SDM for longitudinal scale-proportional factor estimated from step 1 .
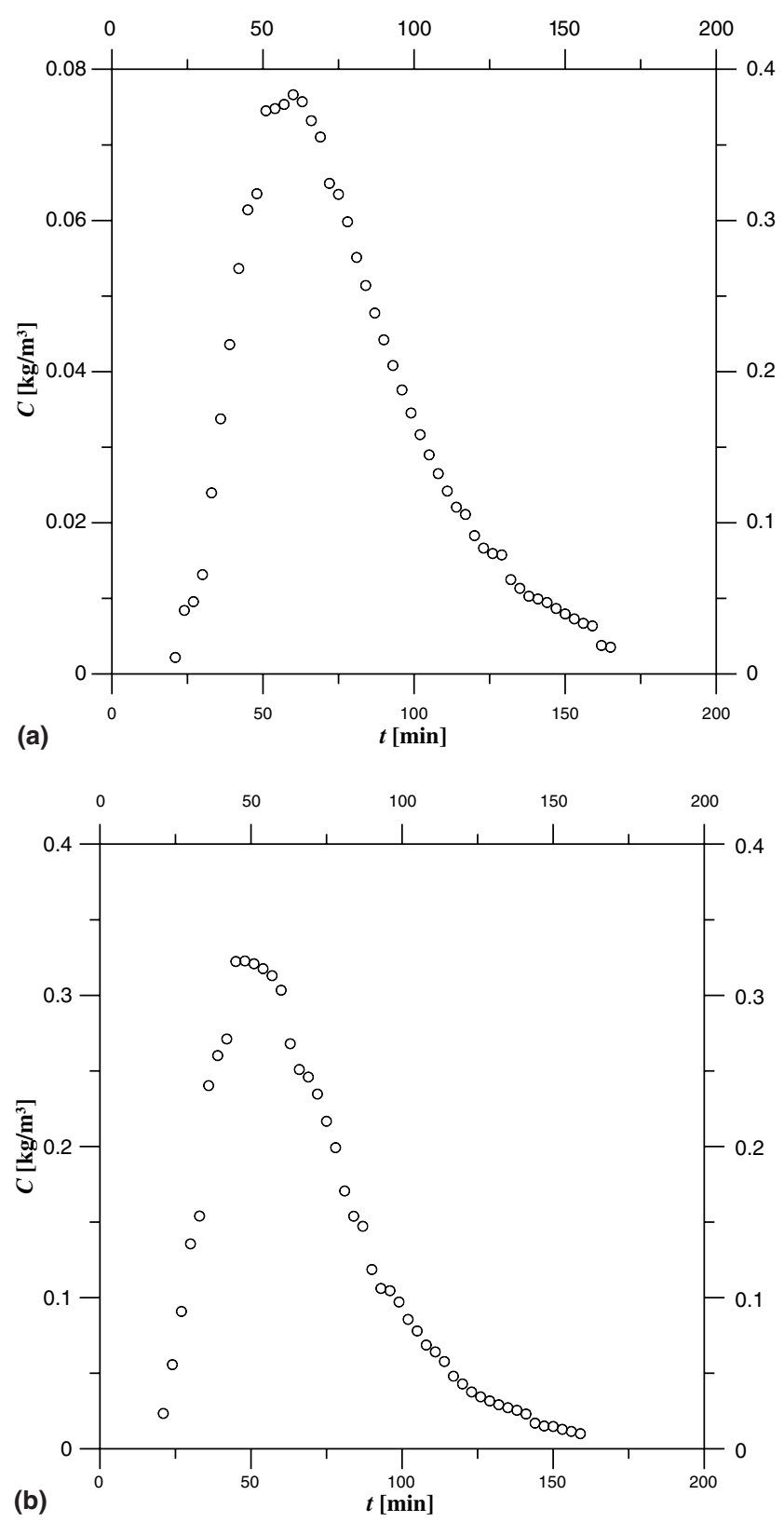

Fig. 8. Hypothetical concentration data at: (a) extraction well and (b) observation well for a convergent tracer test. 


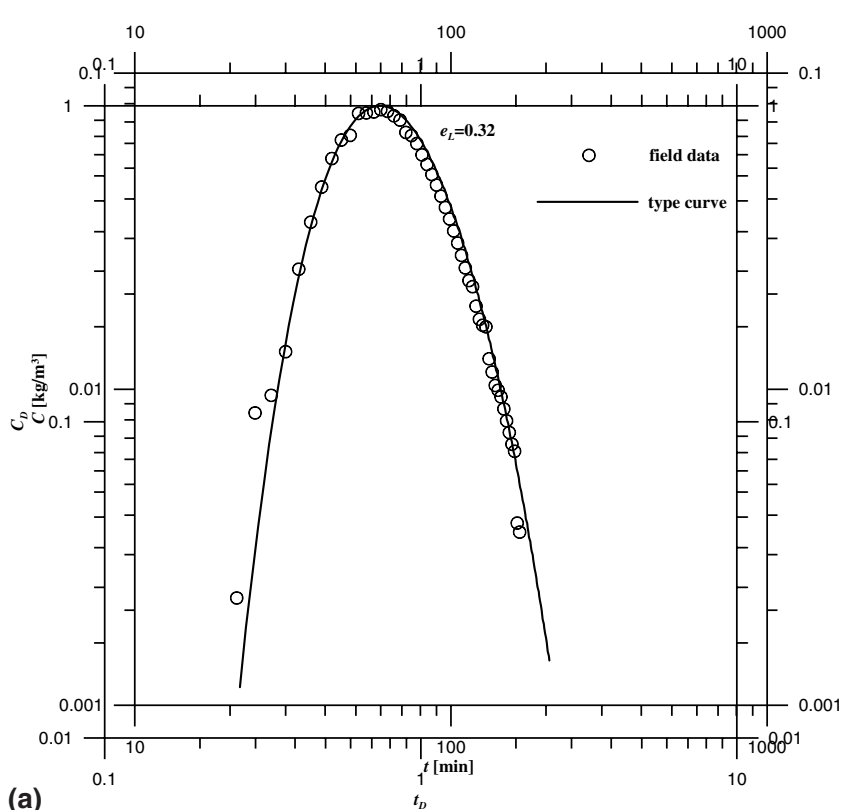

(a)

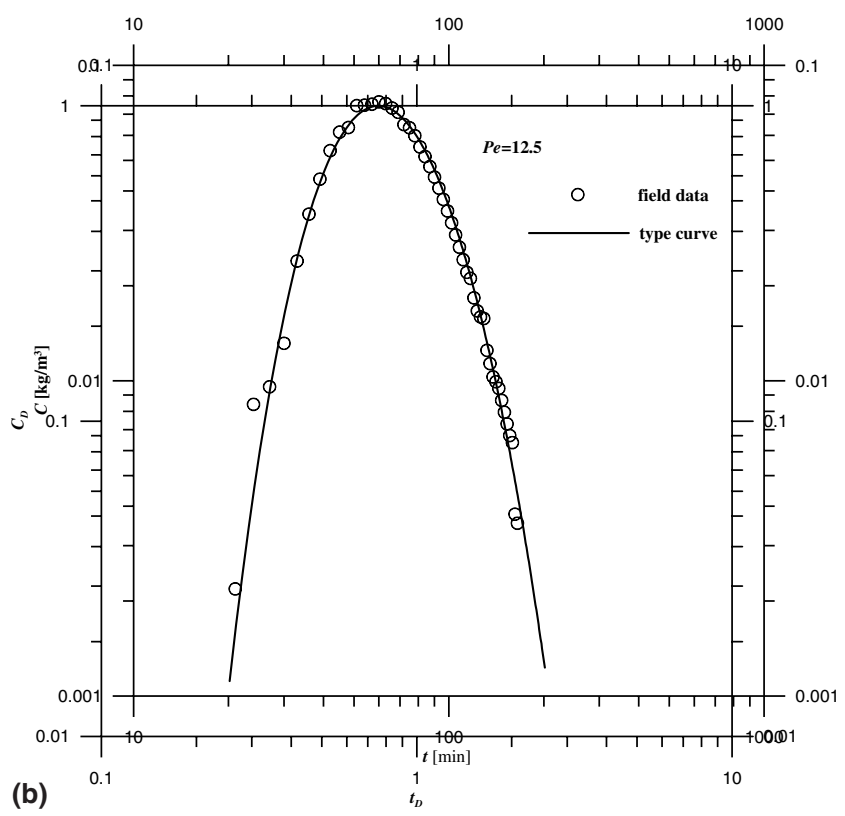

Fig. 9. Hypothetical concentration data superimposed on the type curves at extraction well from (a) the scale-dependent dispersivity model (SDM) and (b) the constant dispersivity model (CDM).

(c) The experimental breakthrough curve is matched against the theoretical breakthrough curves and the dimensionless transverse/longitudinal dispersivity ratio is obtained.

(d) The transverse dispersivity/distance ratio is calculated from

$$
e_{\mathrm{T}}=e_{\mathrm{L}} X_{\mathrm{s}}
$$

Next, a hypothetical example is presented to illustrate the evaluation of longitudinal and transverse dispersivi-
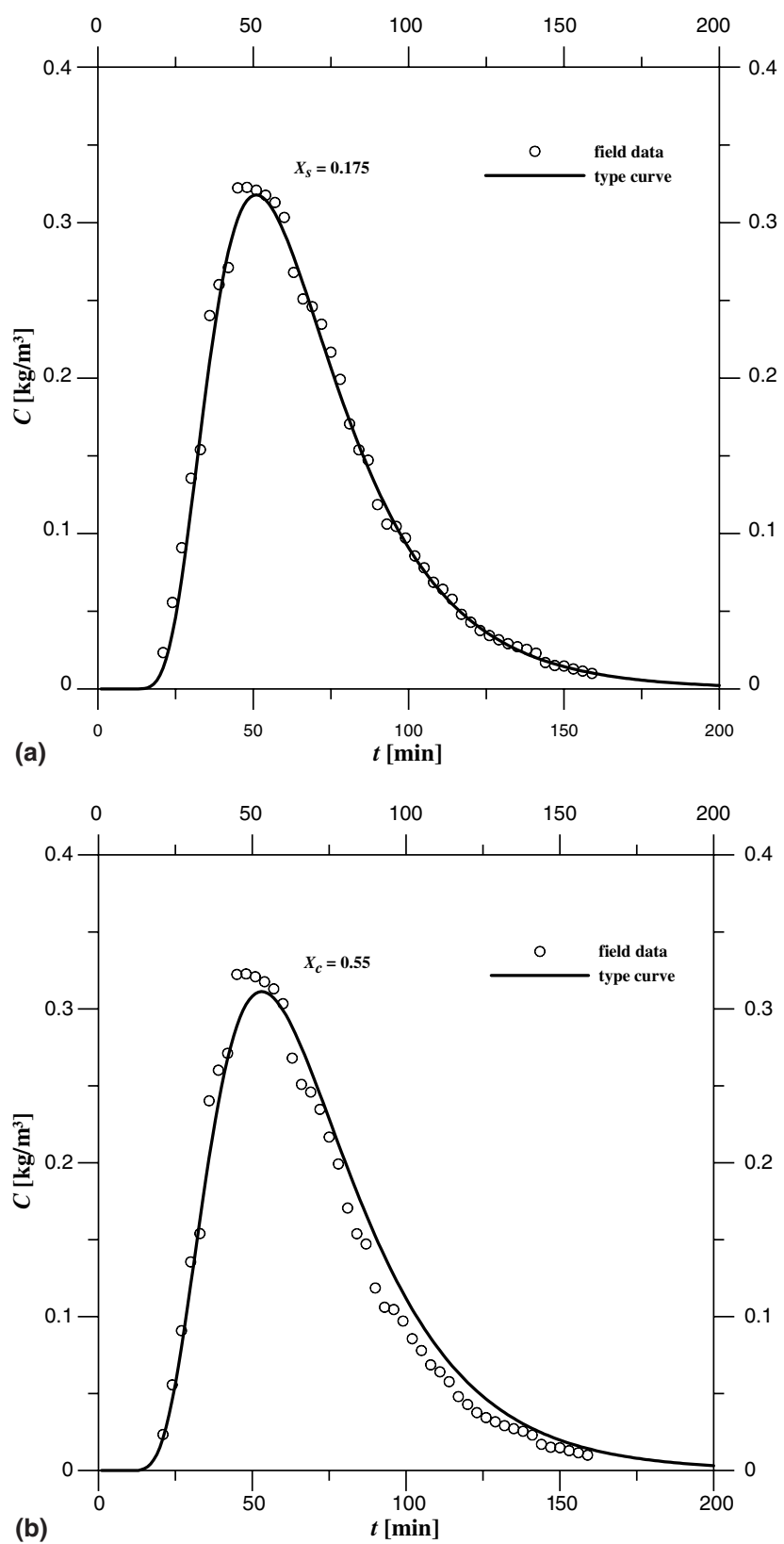

Fig. 10. Hypothetical concentration data superimposed on the theoretical breakthrough curves at observation well from (a) the scaledependent dispersivity model (SDM) and (b) the constant dispersivity model (CDM).

ties/distance ratios according to proposed method for tracer test in a radially convergent flow field with scale-dependent dispersion.

Consider a hypothetical tracer test, the distance between the extraction well and the injection well is $5 \mathrm{~m}$. The concentrations are measured at the extraction well and a observation well located at $r=1 \mathrm{~m}, \theta=\pi$. Fig. $8 \mathrm{a}$ and $\mathrm{b}$ presented the hypothetical concentration data at the extraction well and observation well. The hypothetical concentration data are fitted against the type curve obtained from SDM and CDM according 
to the method proposed in this study and Chen et al. [2] respectively. Fig. 9a and $\mathrm{b}$ present the matches of field data with type curve at the extraction well using SDM and CDM. The longitudinal dispersivity/distance ratio is estimated to be 0.32 using SDM. The determined value of Peclet number is 12.5 and the constant longitudinal dispersivity value of $0.4 \mathrm{~m}$ from curve-fitting using CDM. The yielded longitudinal dispersivity/distance ratio using SDM or longitudinal dispersivity using $\mathrm{CDM}$ are then served as the input parameters to generated the theoretical breakthrough curves at observation well for different dimensionless dispersivity ratios using SDM and CDM. As concentration data are satisfactory matched with the generated theoretical breakthrough curves, the dimensionless dispersivity ratios can be obtained as shown in Fig. 10a and b. The estimated dimensionless dispersivity ratios are 0.175 and 0.55 using SDM and CDM, respectively.

Theoretically, one extraction well and an observation well are enough to determine longitudinal and transverse dispersivity/distance ratios. If concentration data are available at several intermediate wells, they can be used to crosscheck the obtained parameters or to apparently validate the dispersivity/distance relationship.

\section{Conclusion}

This work has presented a mathematical model for analysis of non-axisymmetrical solute transport in a radially convergent flow field with scale-dependent dispersion. The proposed model assumes that both the longitudinal and transverse dispersivities are the linear functions of solute travel distance from the injected source. The Laplace transform finite difference technique is applied to solve the two-dimensional scaledependent advection-dispersion equation in cylindrical coordinates. Comparisons between breakthrough curves from the constant dispersivity model and the scaledependent dispersivity model show the constant dispersivity model can produce the same curves of averaging concentration only at observation distance near the extraction well as well as those from the scale-dependent dispersivity model because that the dispersivity used in the constant dispersivity model are obtained by fitting breakthrough curves from the constant dispersivity model against that from the scale-dependent dispersivity model at extraction. Additionally, dimensionless longitudinal/transverse dispersivity ratio from the inverse approach uses the constant dispersivity model may produce parameters that are significantly different from that using the scale-dependent dispersivity model. A curve-fitting method involving curves is proposed to determine the longitudinal/transverse dispersivity ratios. The proposed model provides a means for obtaining longitudinal and transverse scale-proportional factors by applying it to a field case if the test site obeys the linearly scale-dependent assumption.

\section{Appendix A}

The LTFD solution of the governing Eq. (16), subject to initial and boundary conditions (17)-(23) is derived.

First, by performing the Laplace transform of Eqs. (16) and (17) and its associated boundary conditions (18)-(23) with respect to $t_{\mathrm{D}}$ yields

$$
\begin{aligned}
& e_{\mathrm{L}}\left(1-r_{\mathrm{D}}\right) \frac{\partial^{2} G}{\partial^{2} r_{\mathrm{D}}}+\left(1-e_{\mathrm{T}}\right) \frac{\partial G}{\partial r_{\mathrm{D}}}+\frac{e_{\mathrm{T}}\left(1-r_{\mathrm{D}}\right)}{r_{\mathrm{D}}^{2}} \frac{\partial^{2} G}{\partial \theta^{2}} \\
& \quad=\frac{2 r_{\mathrm{D}} R}{\left(1-r_{\mathrm{wD}}^{2}\right)} s G \\
& \frac{\partial G\left(r_{\mathrm{D}}, \theta, s\right)}{\partial r_{\mathrm{D}}}=0 \quad \text { at } r_{\mathrm{D}}=r_{\mathrm{wD}} \\
& \bar{G}\left(r_{\mathrm{D}}, \theta, s\right)= \begin{cases}C_{\mathrm{I}}(s) & \pi-\delta<|\theta|<\pi \\
0 & 0<|\theta|<\pi-\delta\end{cases} \\
& -G_{\mathrm{I}}(s)=\mu_{\mathrm{I}}\left[s G_{\mathrm{I}}(s)-C_{0}\right] \\
& \frac{\partial G\left(r_{\mathrm{D}}, \theta, s\right)}{\partial \theta}=0 \quad \text { at } \theta=0 \\
& \frac{\partial G\left(r_{\mathrm{D}}, \theta, s\right)}{\partial \theta}=0 \quad \text { at } \theta=\pi
\end{aligned}
$$

where $s$ denotes the Laplace transform parameters and $G$ represents the Laplace transform of $C$, as defined by

$G\left(r_{\mathrm{D}}, \theta, s\right)=\int_{0}^{\infty} C\left(r_{\mathrm{D}}, \theta, t_{\mathrm{D}}\right) \mathrm{e}^{-s t_{\mathrm{D}}} \mathrm{d} t_{\mathrm{D}}$

$G_{\mathrm{I}}(s)=\int_{0}^{\infty} C_{\mathrm{I}}\left(t_{\mathrm{D}}\right) \mathrm{e}^{-s t_{\mathrm{D}}} \mathrm{d} t_{\mathrm{D}}$

Taking the finite Fourier cosine transform with respect to $\theta$ of Eqs. (A.1)-(A.6) yields:

$$
\begin{gathered}
e_{\mathrm{L}}\left(1-r_{\mathrm{D}}\right) \frac{\mathrm{d}^{2} W}{\mathrm{~d}^{2} r_{\mathrm{D}}}+\left(1-e_{\mathrm{L}}\right) \frac{\mathrm{d} W}{\mathrm{~d} r_{\mathrm{D}}} \\
-\left[\frac{e_{\mathrm{T}}\left(1-r_{\mathrm{D}}\right)}{r_{\mathrm{D}}^{2}} n^{2}+\frac{2 r_{\mathrm{D}} R}{\left(1-r_{\mathrm{wD}}^{2}\right)} s\right] W=0 \\
\frac{\mathrm{d} W\left(r_{\mathrm{D}}, n, s\right)}{\mathrm{d} r_{\mathrm{D}}}=0 \\
W\left(r_{\mathrm{D}}, n, s\right)= \begin{cases}G_{\mathrm{I}}(s) \delta & \text { at } r_{\mathrm{D}}=r_{\mathrm{wD}} \\
G_{\mathrm{I}}(s)\left[\frac{(-1)^{n} \sin (n \delta)}{n}\right] & n=1,2,3\end{cases}
\end{gathered}
$$

and

$G_{\mathrm{I}}(s)=\frac{\mu_{\mathrm{I}} C_{0}}{\mu_{\mathrm{I}} s+1}$

where $n$ denotes the finite Fourier cosine transform parameter and $W$ represents the finite Fourier cosine transform of $G$, as defined by

$$
W\left(r_{\mathrm{D}}, n, s\right)=\int_{0}^{\pi} G\left(r_{\mathrm{D}}, \theta, s\right) \cos (n \theta) \mathrm{d} \theta
$$


Such a transform is advantageous in that the inversion is directly given by the following formula [16]:

$G\left(r_{\mathrm{D}}, \theta, s\right)=\frac{1}{\pi} W\left(r_{\mathrm{D}}, 0, s\right)+\frac{2}{\pi} \sum_{n=1}^{\infty} W\left(r_{\mathrm{D}}, n, s\right) \cos (n \theta)$

A finite difference method is applied by discretizing the radial distance of the transformed ordinary differential equation (A.9). The advection term is approximated by the upwind difference formulae. Substituting the difference formulae into the transformed partial differential equation yields an algebraic equation as follows:

$$
\begin{gathered}
e_{\mathrm{L}}\left[1-\left(r_{\mathrm{D}}\right)_{i}\right] \frac{W_{i+1}-2 W_{i}+W_{i-1}}{\left(\Delta r_{\mathrm{D}}\right)^{2}}+\left(1-e_{\mathrm{L}}\right) \frac{W_{i+1}-W_{i}}{\Delta r_{\mathrm{D}}} \\
-\left\{\frac{e_{\mathrm{T}}\left[1-\left(r_{\mathrm{D}}\right)_{i}\right]}{\left(r_{\mathrm{D}}\right)_{i}^{2}} n^{2}+\frac{2\left(r_{\mathrm{D}}\right)_{i} R}{\left(1-r_{\mathrm{wD}}^{2}\right)} s\right\} W_{i}=0
\end{gathered}
$$

The finite difference equation in the Laplace domain is rearranged as follows:

$$
\begin{aligned}
& A_{1} W_{i+1}+A_{2} W_{i}+A_{3} W_{i-1}=A_{4} \\
& A_{1}=\frac{e_{\mathrm{L}}\left[1-\left(r_{\mathrm{D}}\right)_{i}\right]}{\left(\Delta r_{\mathrm{D}}\right)^{2}}+\frac{\left(1-e_{\mathrm{L}}\right)}{\Delta r_{\mathrm{D}}} \\
& A_{2}=\frac{-2 e_{\mathrm{L}}\left[1-\left(r_{\mathrm{D}}\right)_{i}\right]}{\left(\Delta r_{\mathrm{D}}\right)^{2}}-\frac{1-e_{\mathrm{L}}}{\Delta r_{\mathrm{D}}} \\
& -\left\{\frac{e_{\mathrm{T}}\left[1-\left(r_{\mathrm{D}}\right)_{i}\right]}{\left(r_{\mathrm{D}}\right)_{i}^{2}} n^{2}+\frac{2\left(r_{\mathrm{D}}\right)_{i} R}{\left(1-r_{\mathrm{wD}}^{2}\right)} s\right\} \\
& A_{3}=\frac{e_{\mathrm{L}}\left[1-\left(r_{\mathrm{D}}\right)_{i}\right]}{\left(\Delta r_{\mathrm{D}}\right)^{2}} \\
& A_{4}=0
\end{aligned}
$$

Written in matrix notation, the finite difference system of simultaneous Eq. (A.15) becomes

$[\Omega][W]=[D]$

where $[\Omega]$ denotes the coefficient matrix; $[W]$ represents the vector of the unknown transformed concentration, and $[D]$ is the known right-hand side vector. The system of algebraic equations represented by Eq. (A.16), can be solved using direct Gaussian elimination or other solvers (e.g., iterative types) to yield a Laplace transformed concentration at the node points. Additionally, a FORTRAN subroutine DLSACB is readily available [17]. The solutions in original domain $C\left(r_{\mathrm{D}}, \theta, t_{\mathrm{D}}\right)$ are the Laplace and finite Fourier cosine inversion of $W\left(r_{\mathrm{D}}, n, s\right)$. For convenience, the finite Fourier cosine transform is performed first. Also, the Laplace inverse of $W\left(r_{\mathrm{D}}, n, s\right)$ must be determined numerically. A FORTRAN subrou- tine DINLAP/INLAP [17] based on the De Hoog et al. [4] algorithm, is employed to perform the Laplace inversion.

\section{References}

[1] Aral MM, Liao B. Analytical solutions for two-dimensional transport equation with time-dependent dispersion coefficients. J Hydrol Eng 1996;1:20-32.

[2] Chen JS, Chen CS, Gau HS, Liu CW. A two-well method to evaluate transverse dispersivity for tracer tests in a radially convergent flow field. J Hydrol 1999;223(3-4):175-97.

[3] Chen JS, Liu CW, Hsu HT, Liao CM. A Laplace transformed power series solution for solute transport in a convergent flow field with scale-dependent dispersion. Water Resour Res 2003;39(8). doi:10.1029/2003WR002299.

[4] De Hoog FR, Knight JH, Stokes AN. An improved method of Laplace transforms using a Fourier series approximation. SIAMJ Sci Stat Comput 1982;3(3):357-66.

[5] Gelhar LW. Stochastic subsurface hydrology from theory to applications. Water Resour Res 1986;22(9):135S-45S.

[6] Hunt B. Contaminant source solutions with scale-dependent dispersivity. J Hydrol Eng 1998;3(4):268-75.

[7] Hunt B. Scale-dependent dispersion from a pit. J Hydrol Eng 2002;7(4):168-74.

[8] Jayawardena AW, Lui PH. Numerical solution of the dispersion equation using a variable dispersion coefficient; method and applications. Hydrol Sci J 1984;29(3):293-309.

[9] Kocabas I, Islam MR. Concentration and temperature transients in heterogeneous porous media. Part II: Radial transport. J Petrol Sci Eng 2000;26:221-33.

[10] Logan JD. Solute transport in porous media with scale-dependent dispersion and periodic boundary conditions. J Hydrol 1996;184:261-76.

[11] Mishra S, Parker JC. Analysis of solute transport with a hyperbolic scale dependent dispersion model. Hydrol Proc 1989;4(1):45-57.

[12] Moench AF. Convergent radial dispersion: a Laplace transform solution for aquifer tracer testing. Water Resour Res 1989;25(3):439-47.

[13] Pang L, Hunt B. Solutions and verification of a scale-dependent dispersion model. J Contam Hydrol 2001;53:21-39.

[14] Pickens JF, Grisak GE. Scale-dependent dispersion in a stratified granular aquifer. Water Resour Res 1981;17(4):1191-211.

[15] Pickens JF, Grisak GE. Modelling of scale-dependent dispersion in a hydrogeological system. Water Resour Res 1981;17(6): 1701-11.

[16] Sneddon IN. The Use of Integral Transform. New York: McGraw-Hill; 1972.

[17] IMSL User's Manual, vol. 1. Houston, TX: Visual Numerical Inc.; 1994. p. 159-61 and p. 827-30.

[18] Yates SR. An analytical solution for one-dimensional transport in heterogeneous porous media. Water Resour Res 1990;26(10): 2331-8.

[19] Yates SR. An analytical solution for one-dimensional transport in heterogeneous porous media with an exponential dispersion function. Water Resour Res 1992;28(18):2149-54.

[20] Zlotnik VA, Logan JD. Boundary conditions for convergent radial tracer tests and the effect of well bore mixing volume. Water Resour Res 1996;32(7):2323-8. 